\title{
A note on statistical convergence on time scale
}

\author{
M Seyyit Seyyidoglu and N Özkan Tan*
}

${ }^{\text {"Correspondence: }}$ nozkan.tan@usak.edu.tr

Faculty of Sciences and Arts, Department of Mathematics, Usak University, 1 Eylul Campus, Usak, 64200, Turkey

\begin{abstract}
In the present paper, we will give some new notions, such as $\Delta$-convergence and $\Delta$-Cauchy, by using the $\Delta$-density and investigate their relations. It is important to say that the results presented in this work generalize some of the results mentioned in the theory of statistical convergence.
\end{abstract}

MSC: $34 \mathrm{~N} 05$

Keywords: time scale; Lebesgue $\Delta$-measure; statistical convergence

\section{Introduction and background}

In [1] Fast introduced an extension of the usual concept of sequential limits which he called statistical convergence. In [2] Schoenberg gave some basic properties of statistical convergence. In [3] Fridy introduced the concept of a statistically Cauchy sequence and proved that it is equivalent to statistical convergence.

The theory of time scales was introduced by Hilger in his PhD thesis supervised by Auldbach [4] in 1988. The measure theory on time scales was first constructed by Guseinov [5], and then further studies were performed by Cabada-Vivero [6] and Rzezuchowski [7]. In [8] Deniz-Ufuktepe define Lebesgue-Stieltjes $\Delta$ and $\nabla$-measures, and by using these measures, they define an integral adapted to a time scale, specifically Lebesgue-Steltjes $\Delta$-integral.

A time scale $\mathbb{T}$ is an arbitrary nonempty closed subset of the real numbers $\mathbb{R}$. The time scale $\mathbb{T}$ is a complete metric space with the usual metric. We assume throughout the paper that a time scale $\mathbb{T}$ has the topology that it inherits from the real numbers with the standard topology.

For $t \in \mathbb{T}$, we define the forward jump operator $\sigma: \mathbb{T} \rightarrow \mathbb{T}$ by

$$
\sigma(t):=\inf \{s \in \mathbb{T}: s>t\}
$$

In this definition, we put $\inf \emptyset=\sup \mathbb{T}$.

For $a, b \in \mathbb{T}$ with $a \leq b$, we define the interval $[a, b]$ in $\mathbb{T}$ by

$$
[a, b]=\{t \in \mathbb{T}: a \leq t \leq b\}
$$

Open intervals and half-open intervals etc. are defined accordingly.

Let $\mathbb{T}$ be a time scale. Denote by $\mathcal{S}$ the family of all left-closed and right-open intervals of $\mathbb{T}$ of the form $[a, b)=\{t \in \mathbb{T}: a \leq t<b\}$ with $a, b \in \mathbb{T}$ and $a \leq b$. The interval $[a, a)$ is

\section{望 Springer}

(c) 2012 Seyyidoglu and Tan; licensee Springer. This is an Open Access article distributed under the terms of the Creative Commons Attribution License (http://creativecommons.org/licenses/by/2.0), which permits unrestricted use, distribution, and reproduction in any medium, provided the original work is properly cited. 
understood as an empty set. $\mathcal{S}$ is a semiring of subsets of $\mathbb{T}$. Obviously, the set function $m: \mathcal{S} \rightarrow[0, \infty]$ defined by $m([a, b))=b-a$ is a countably additive measure. An outer measure $m^{*}: \mathcal{P}(\mathbb{T}) \rightarrow[0, \infty]$ generated by $m$ is defined by

$$
m^{*}(A)=\inf \left\{\sum_{n=1}^{\infty} m\left(A_{n}\right):\left(A_{n}\right) \text { is a sequence of } \mathcal{S} \text { with } A \subset \bigcup_{n=1}^{\infty} A_{n}\right\} .
$$

If there is no sequence $\left(A_{n}\right)$ of $\mathcal{S}$ such that $A \subset \bigcup_{n=1}^{\infty} A_{n}$, then we let $m^{*}(A)=\infty$. We define the family $\mathcal{M}\left(m^{*}\right)$ of all $m^{*}$-measurable subsets of $\mathbb{T}$, i.e.,

$$
\mathcal{M}\left(m^{*}\right)=\left\{E \subset \mathbb{T}: m^{*}(A)=m^{*}(A \cap E)+m^{*}\left(A \cap E^{c}\right) \text { for all } A \subset \mathbb{T}\right\}
$$

The collection $\mathcal{M}\left(m^{*}\right)$ of all $m^{*}$-measurable sets is a $\sigma$-algebra, and the restriction of $m^{*}$ to $\mathcal{M}\left(m^{*}\right)$, which we denote by $\mu_{\Delta}$, is a countably additive measure on $\mathcal{M}\left(m^{*}\right)$. We call this measure $\mu_{\Delta}$, which is the Carathéodory extension of the set function $m$ associated with the family $\mathcal{S}$, the Lebesgue $\Delta$-measure on $\mathbb{T}$.

We call $f: \mathbb{T} \rightarrow \mathbb{R}$ a measurable function, if $f^{-1}(\mathcal{O}) \in \mathcal{M}\left(m^{*}\right)$ for every open subset of $\mathcal{O}$ of $\mathbb{R}$.

Theorem 1 (see [5]) For each $t_{0}$ in $\mathbb{T}-\{\max \mathbb{T}\}$, the single point set $\left\{t_{0}\right\}$ is $\Delta$-measurable, and its $\Delta$-measure is given by

$$
\mu_{\Delta}\left(\left\{t_{0}\right\}\right)=\sigma\left(t_{0}\right)-t_{0}
$$

Theorem 2 (see [5]) If $a, b \in \mathbb{T}$ and $a \leq b$, then

$$
\mu_{\Delta}([a, b))=b-a, \quad \mu_{\Delta}((a, b))=b-\sigma(a) .
$$

If $a, b \in \mathbb{T}-\{\max \mathbb{T}\}$ and $a \leq b$, then

$$
\mu_{\Delta}((a, b])=\sigma(b)-\sigma(a), \quad \mu_{\Delta}([a, b])=\sigma(b)-a .
$$

It can easily be seen from Theorem 1 that the measure of a subset of $\mathbb{N}$ is equal to its cardinality.

\section{$2 \Delta$-density, $\Delta$-convergence, $\Delta$-Cauchy}

It is well known that the notions of statistical convergence and statistical Cauchy are closely related to the density of the subset of $\mathbb{N}$. In the present section, first of all, we will define the density of the subset of the time scale. By using this definition, we will define $\Delta$-convergence and $\Delta$-Cauchy for a real valued function defined on the time scale. Then we will show that these notions are equivalent.

Throughout this paper, we consider the time scales which are unbounded from above and have a minimum point.

Let $A$ be a $\Delta$-measurable subset of $\mathbb{T}$ and let $a=\min \mathbb{T}$. $\Delta$-density of $A$ in $\mathbb{T}$ (or briefly $\Delta$-density of $A$ ) is defined by

$$
\delta_{\Delta}(A)=\lim _{t \rightarrow \infty} \frac{\mu_{\Delta}(A(t))}{\sigma(t)-a}
$$


(if this limit exists), where

$$
A(t)=\{s \in A: s \leq t\}
$$

and $t \in \mathbb{T}$.

From the identity $A(t)=A \cap[a, t]$, the measurability of $A$ implies the measurability of $A(t)$.

If $f: \mathbb{T} \rightarrow \mathbb{R}$ is a function such that $f(t)$ satisfies the property $P$ for all $t$ except a set of $\Delta$-density zero, then we say that $f(t)$ satisfies $P$ for ' $\Delta$-almost all $t$ ', and we abbreviate this by ' $\Delta$-a.a. $t$ '. Let

$$
\begin{aligned}
& \mathcal{M}_{d}:=\{A \in \mathbb{T}: \Delta \text {-density of } A \text { exists in } \mathbb{T}\} \\
& \mathcal{M}_{d}^{0}:=\left\{A \in \mathbb{T}: \delta_{\Delta}(A)=0\right\} .
\end{aligned}
$$

\section{Lemma 1}

(i) If $A, B \in \mathcal{M}_{d}$ and $A \subset B$, then $\delta_{\Delta}(A) \leq \delta_{\Delta}(B)$.

(ii) If $A \in \mathcal{M}_{d}$, then $0 \leq \delta_{\Delta}(A) \leq 1$.

(iii) $\mathbb{T} \in \mathcal{M}_{d}$ and $\delta_{\Delta}(\mathbb{T})=1$.

(iv) If $A \in \mathcal{M}_{d}$, then $A^{c} \in \mathcal{M}_{d}$ and $\delta_{\Delta}(A)+\delta_{\Delta}\left(A^{c}\right)=1$.

(v) If $A, B \in \mathcal{M}_{d}$ and $A \subset B$, then $B-A \in \mathcal{M}_{d}$ and $\delta_{\Delta}(B-A)=\delta_{\Delta}(B)-\delta_{\Delta}(A)$.

(vi) If $A_{1}, A_{2}, \ldots, A_{n}$ is a mutually disjoint sequence in $\mathcal{M}_{d}$, then $\bigcup_{k=1}^{n} A_{k} \in \mathcal{M}_{d}$ and

$$
\delta_{\Delta}\left(\bigcup_{k=1}^{n} A_{k}\right)=\sum_{k=1}^{n} \delta_{\Delta}\left(A_{k}\right)
$$

(vii) If $A_{1}, A_{2}, \ldots, A_{n}$ in $\mathcal{M}_{d}$ and $\bigcup_{k=1}^{n} A_{k} \in \mathcal{M}_{d}$, then

$$
\delta_{\Delta}\left(\bigcup_{k=1}^{n} A_{k}\right) \leq \sum_{k=1}^{n} \delta_{\Delta}\left(A_{k}\right)
$$

(viii) If $A$ is a measurable set and $B \in \mathcal{M}_{d}^{0}$ with $A \subset B$, then $A \in \mathcal{M}_{d}^{0}$.

(ix) If $A_{1}, A_{2}, \ldots, A_{n}$ in $\mathcal{M}_{d}^{0}$, then $\bigcup_{k=1}^{n} A_{k}, \bigcap_{k=1}^{n} A_{k} \in \mathcal{M}_{d}^{0}$.

(x) Every bounded measurable subset of $\mathbb{T}$ belongs to $\mathcal{M}_{d}^{0}$.

(xi) If $A \in \mathcal{M}_{d}^{0}$ and $B \in \mathcal{M}_{d}$, then $\delta_{\Delta}(A \cup B)=\delta_{\Delta}(B)$.

Proof (i) Let $A, B \in \mathcal{M}_{d}$ and $A \subset B$. Clearly, $A(t) \subset B(t)$, and since $\mu_{\Delta}$ is a measure function, one has $\mu_{\Delta}(A) \leq \mu_{\Delta}(B)$. Thus, we have

$$
\delta_{\Delta}(A)=\lim _{t \rightarrow \infty} \frac{\mu_{\Delta}(A(t))}{\sigma(t)-a} \leq \lim _{t \rightarrow \infty} \frac{\mu_{\Delta}(B(t))}{\sigma(t)-a}=\delta_{\Delta}(B) .
$$

(ii) Note that $A(t) \subset[a, t]$. The required inequalities follow from the following inequalities:

$$
0 \leq \frac{\mu_{\Delta}(A(t))}{\sigma(t)-a} \leq \frac{\mu_{\Delta}([a, t])}{\sigma(t)-a}=\frac{\sigma(t)-a}{\sigma(t)-a}=1 .
$$


(iii) It is clear that $\mathbb{T}$ is measurable. The $\Delta$-density of $\mathbb{T}$ is obtained from the following equalities:

$$
\delta_{\Delta}(\mathbb{T})=\lim _{t \rightarrow \infty} \frac{\mu_{\Delta}(\mathbb{T}(t))}{\sigma(t)-a}=\lim _{t \rightarrow \infty} \frac{\mu_{\Delta}([a, t])}{\sigma(t)-a}=1 .
$$

(iv) Since $A$ is measurable, so is $A^{c}$, namely $A^{c}(t)=\left\{s \leq t: s \in A^{c}\right\}$ is measurable. On the other hand, $A(t) \cup A^{c}(t)=[a, t]$ and

$$
1=\frac{\mu_{\Delta}([a, t])}{\sigma(t)-a}=\frac{\mu_{\Delta}(A(t))}{\sigma(t)-a}+\frac{\mu_{\Delta}\left(A^{c}(t)\right)}{\sigma(t)-a}
$$

imply that the required statement holds as $t \rightarrow \infty$.

(v) Since $A$ and $B$ are measurable, so are $B-A$. The statement can be easily shown by considering $A(t) \cup(B-A)(t) \cup B^{c}(t)=[a, t]$.

(vi) Since the $\Delta$-density of for each subset $A_{k}$ exists, one can write

$$
\begin{aligned}
\sum_{k=1}^{n} \delta_{\Delta}\left(A_{k}\right) & =\sum_{k=1}^{n} \lim _{t \rightarrow \infty} \frac{\mu_{\Delta}\left(A_{k}(t)\right)}{\sigma(t)-a} \\
& =\lim _{t \rightarrow \infty} \sum_{k=1}^{n} \frac{\mu_{\Delta}\left(A_{k}(t)\right)}{\sigma(t)-a} \\
& =\lim _{t \rightarrow \infty} \frac{\mu_{\Delta}\left(\bigcup_{k=1}^{n} A_{k}(t)\right)}{\sigma(t)-a} \\
& =\lim _{t \rightarrow \infty} \frac{\mu_{\Delta}\left(\left(\bigcup_{k=1}^{n} A_{k}\right)(t)\right)}{\sigma(t)-a} \\
& =\delta_{\Delta}\left(\bigcup_{k=1}^{n} A_{k}\right) .
\end{aligned}
$$

(vii) The proof is similar to that of the previous proof.

(viii) It can be easily seen from (i).

(ix) Considering $\delta_{\Delta}\left(A_{k}\right)=0$ for $k=1,2, \ldots, n$ and (vii), one can obtain $\bigcup_{k=1}^{n} A_{k} \in \mathcal{M}_{d}^{0}$. And $\bigcap_{k=1}^{n} A_{k} \in \mathcal{M}_{d}^{0}$ can be obtained from (viii).

(x) Let $A$ be a bounded set. For a sufficiently large $M \in \mathbb{T}$, we can write $A \subset[a, M]$. Then one has

$$
0 \leq \frac{\mu_{\Delta}(A(t))}{\sigma(t)-a} \leq \frac{\mu_{\Delta}([a, M])}{\sigma(t)-a} \rightarrow 0 \quad(t \rightarrow \infty)
$$

which implies that $\delta_{\Delta}(A)=0$.

(xi) (i) and (vii) yield $\delta_{\Delta}(B) \leq \delta_{\Delta}(A \cup B) \leq \delta_{\Delta}(A)+\delta_{\Delta}(B)=\delta_{\Delta}(B)$. This completes the proof.

It is clear that the family $\mathcal{M}_{d}^{0}$ is a ring of subsets of $\mathbb{T}$. According to (iv), the $\Delta$-density of the complement of a subset whose $\Delta$-density is 0 is equal to $1, \mathcal{M}_{d}^{0}$ is not closed under the operation complement. So, it is not an algebra. Note that the $\Delta$-density of a subset of $\mathbb{N}$ is equal to its natural density. 
Example 1 Let $\mathbb{T}=[0, \infty), l$ and $r$ be arbitrary two positive real numbers. Let also $A=$ $\bigcup_{n=0}^{\infty} A_{n}$, where $A_{n}=[n l+n r,(n+1) l+n r]$. According to Lemma $1(\mathrm{x})$, each $A_{n}$ is bounded and so $\delta_{\Delta}\left(A_{n}\right)=0$. In addition, let $A(t)$ be defined as in (2.1), we have

$$
\mu_{\Delta}(A(t))= \begin{cases}t-n r, & n l+n r \leq t \leq(n+1) l+n r, \\ (n+1) l, & (n+1) l+n r \leq t \leq(n+1) l+(n+1) r\end{cases}
$$

$(n=0,1,2, \ldots)$, and hence

$$
\delta_{\Delta}(A)=\lim _{t \rightarrow \infty} \frac{\mu_{\Delta}(A(t))}{\sigma(t)-a}=\lim _{t \rightarrow \infty} \frac{\mu_{\Delta}(A(t))}{t-0}=\frac{l}{l+r} .
$$

Note that since

$$
\frac{l}{l+r}=\delta_{\Delta}(A)>\sum_{n=0}^{\infty} \delta_{\Delta}\left(A_{n}\right)=0
$$

$\delta_{\Delta}$ does not define a measure.

Example 2 Let $\mathbb{T}=\mathbb{N}$. The $\Delta$-density of $A=\{2,4,6, \ldots\}$ in $\mathbb{N}$ is given by

$$
\delta_{\Delta}(A)=\lim _{t \rightarrow \infty} \frac{\mu_{\Delta}(A(t))}{\sigma(t)-a}=\lim _{t \rightarrow \infty} \frac{[t / 2]}{t}=\frac{1}{2} .
$$

Definition 1 ( $\Delta$-convergence) The function $f: \mathbb{T} \rightarrow \mathbb{R}$ is $\Delta$-convergent to the number $L$ provided that for each $\varepsilon>0$, there exists $K_{\varepsilon} \subset \mathbb{T}$ such that $\delta_{\Delta}\left(K_{\varepsilon}\right)=1$ and $|f(t)-L|<\varepsilon$ holds for all $t \in K_{\varepsilon}$.

We will use notation $\Delta-\lim _{t \rightarrow \infty} f(t)=L$.

Definition 2 ( $\Delta$-Cauchy) The function $f: \mathbb{T} \rightarrow \mathbb{R}$ is $\Delta$-Cauchy provided that for each $\varepsilon>0$, there exists $K_{\varepsilon} \subset \mathbb{T}$ and $t_{0} \in \mathbb{T}$ such that $\delta_{\Delta}\left(K_{\varepsilon}\right)=1$ and $\left|f(t)-f\left(t_{0}\right)\right|<\varepsilon$ holds for all $t \in K_{\varepsilon}$.

Proposition 1 Let $f: \mathbb{T} \rightarrow \mathbb{R}$ be a measurable function. $\Delta-\lim _{t \rightarrow \infty} f(t)=L$ if and only if, for each $\varepsilon>0, \delta_{\Delta}(\{t \in \mathbb{T}:|f(t)-L| \geq \varepsilon\})=0$.

Proof Let $\Delta-\lim _{t \rightarrow \infty} f(t)=L$ and $\varepsilon>0$ be given. In this case, there exists a subset $K_{\varepsilon} \subset \mathbb{T}$ such that $\delta_{\Delta}\left(K_{\varepsilon}\right)=1$ and $|f(t)-L|<\varepsilon$ holds for all $t \in K_{\varepsilon}$. Since $K_{\varepsilon} \subset\{t \in \mathbb{T}:|f(t)-L|<\varepsilon\}$, we obtain $\delta_{\Delta}(\{t \in \mathbb{T}:|f(t)-L|<\varepsilon\})=1$. Hence, we get $\delta_{\Delta}(\{t \in \mathbb{T}:|f(t)-L| \geq \varepsilon\})=0$.

Another case of the proof is straightforward.

Proposition 2 Let $f: \mathbb{T} \rightarrow \mathbb{R}$ be a measurable function. $f$ is $\Delta$-Cauchy if and only if, for each $\varepsilon>0$, there exists $t_{0} \in \mathbb{T}$ such that $\delta_{\Delta}\left(\left\{t \in \mathbb{T}:\left|f(t)-f\left(t_{0}\right)\right| \geq \varepsilon\right\}\right)=0$.

Example 3 Let $\mathbb{I}_{[0, \infty)}$ be irrational numbers and $\mathbb{Q}_{[0, \infty)}$ be rational numbers in $[0, \infty)$. Let us consider the function $f: \mathbb{T}=[0, \infty) \rightarrow \mathbb{R}$ defined as follows:

$$
f(t)= \begin{cases}0, & t \in \mathbb{Q}_{[0, \infty)}, \\ 1, & t \in \mathbb{I}_{[0, \infty)}\end{cases}
$$


Since $\mu_{\Delta}\left(\mathbb{Q}_{[0, \infty)}\right)=0$, the density of the subset $\mathbb{Q}_{[0, \infty)}$ in the time scale $\mathbb{T}$ is zero. This implies that $\delta_{\Delta}\left(\mathbb{I}_{[0, \infty)}\right)=1$. So, for each $\varepsilon>0$ and for all $t \in \mathbb{I}_{[0, \infty)}$, one has $0=|f(t)-1|<\varepsilon$, and as a corollary, we get $\Delta-\lim _{t \rightarrow \infty} f(t)=1$.

Proposition 3 The $\Delta$-limit of a function $f: \mathbb{T} \rightarrow \mathbb{R}$ is unique.

Proof Let $\Delta-\lim _{t \rightarrow \infty} f(t)=L_{1}$ and $\Delta-\lim _{t \rightarrow \infty} f(t)=L_{2}$. Let $\varepsilon>0$ be given. Then there exist subsets $K_{1}, K_{2} \subset \mathbb{T}$ such that for every $t \in K_{1}$ with $\left|f(t)-L_{1}\right|<\varepsilon / 2$ and for every $t \in K_{2}$ with $\left|f(t)-L_{1}\right|<\varepsilon / 2$, where $\delta_{\Delta}\left(K_{1}\right)=1$ and $\delta_{\Delta}\left(K_{2}\right)=1$. From Lemma $1(\mathrm{iv})$, we have $K_{1} \cap K_{2} \neq \emptyset$. Thus, for every $t \in K_{1} \cap K_{2}$, one has

$$
\left|L_{1}-L_{2}\right| \leq\left|f(t)-L_{1}\right|+\left|f(t)-L_{2}\right|<\varepsilon \text {. }
$$

Thus, $L_{1}=L_{2}$.

Proposition 4 If $f, g: \mathbb{T} \rightarrow \mathbb{R}$ with $\Delta-\lim _{t \rightarrow \infty} f(t)=L_{1}$ and $\Delta-\lim _{t \rightarrow \infty} g(t)=L_{2}$, then the following statements hold:

(i) $\Delta-\lim _{t \rightarrow \infty}(f(t)+g(t))=L_{1}+L_{2}$,

(ii) $\Delta-\lim _{t \rightarrow \infty}(c f(t))=c L_{1}(c \in \mathbb{R})$.

Proposition 5 If $f: \mathbb{T} \rightarrow \mathbb{R}$ with $\lim _{t \rightarrow \infty} f(t)=L$, then $\Delta$ - $\lim _{t \rightarrow \infty} f(t)=L$.

Proof Let $\lim _{t \rightarrow \infty} f(t)=L$. In this case, for a given $\varepsilon>0$, we can find a $t_{0} \in \mathbb{T}$ such that $|f(t)-L|<\varepsilon$ holds for every $t>t_{0}$. The set $K_{\varepsilon}=\left\{t \in \mathbb{T}: t>t_{0}\right\}$ is measurable, and from Lemma 1(iv) and (x), one has $\delta_{\Delta}\left(K_{\varepsilon}\right)=1$. By the definition of $\Delta$-convergence, we get $\Delta-\lim _{t \rightarrow \infty} f(t)=L$.

Theorem 3 Let $f: \mathbb{T} \rightarrow \mathbb{R}$ be a measurable function. The following statements are equivalent:

(i) $f$ is $\Delta$-convergent,

(ii) $f$ is $\Delta$-Cauchy,

(iii) There exists a measurable and convergent function $g: \mathbb{T} \rightarrow \mathbb{R}$ such that $f(t)=g(t)$ for $\Delta$-a.a.t.

Proof (i) $\Rightarrow$ (ii): Let $\Delta-\lim _{t \rightarrow \infty} f(t)=L$ and $\varepsilon>0$ be given. Then $|f(t)-L|<\varepsilon / 2$ holds for $\Delta$-a.a. $t$. We can choose $t_{0} \in \mathbb{T}$ such that $\left|f\left(t_{0}\right)-L\right|<\varepsilon / 2$ holds. So,

$$
\left|f(t)-f\left(t_{0}\right)\right| \leq|f(t)-L|+\left|f\left(t_{0}\right)-L\right|<\varepsilon, \quad \Delta \text {-a.a. } t \text {. }
$$

This shows that $f$ satisfies the property of $\Delta$-Cauchy.

(ii) $\Rightarrow$ (iii): We can choose an element $t_{1} \in \mathbb{T}$. We can define an interval $I=\left[f\left(t_{1}\right)-\right.$ $\left.1, f\left(t_{1}\right)+1\right]$ which contains $f(t)$ for $\Delta$-a.a. $t$. By the same method, we can choose an element $t^{*} \in \mathbb{T}$ and define an interval $I^{\prime}=\left[f\left(t^{*}\right)-1 / 2, f\left(t^{*}\right)+1 / 2\right]$ which contains $f(t)$ for $\Delta$-a.a. $t$. We can write

$$
\left\{t \leq s: f(t) \notin I \cap I^{\prime}\right\}=\{t \leq s: f(t) \notin I\} \cup\left\{t \leq s: f(t) \notin I^{\prime}\right\} .
$$


Since $f$ is a measurable function, the two terms that appear on the right-hand side of the last equality are also measurable. By using Lemma 1(vii), we obtain

$$
\begin{aligned}
\delta_{\Delta}\left(\left\{t \in \mathbb{T}: f(t) \notin I \cap I^{\prime}\right\}\right) & \leq \delta_{\Delta}(\{t \in \mathbb{T}: f(t) \notin I\})+\delta_{\Delta}\left(\left\{t \in \mathbb{T}: f(t) \notin I^{\prime}\right\}\right) \\
& =0 .
\end{aligned}
$$

So, $f(t)$ is in the closed interval $I_{1}=I \cap I^{\prime}$ for $\Delta$-a.a. $t$. It is clear that the length of the interval $I_{1}$ is less than or equal to 1 . Now we can choose $t_{2} \in \mathbb{T}$ with $I^{\prime \prime}=\left[f\left(t_{2}\right)-1 / 4, f\left(t_{2}\right)+\right.$ 1/4] containing $f(t)$ for $\Delta$-a.a. $t$. Undoubtedly, the closed interval $I_{2}=I_{1} \cap I^{\prime \prime}$ contains $f(t)$ for $\Delta$-a.a. $t$ and the length of the interval $I_{2}$ is less than or equal to $1 / 2$. With the same procedure, for each $m$, we can obtain a sequence of closed intervals $\left(I_{m}\right)_{m=1}^{\infty}$ such that $I_{m+1} \subset I_{m}$ and the length of each interval $I_{m}$ is less than or equal to $2^{1-m}$. Moreover, $f(t) \in I_{m}$ for $\Delta$-a.a. $t$. From the properties of the intersection of closed intervals, there exists a real number $\lambda$ such that $\bigcap_{m=1}^{\infty} I_{m}=\{\lambda\}$. Since the $\Delta$-density of the set on which $f(t) \notin I_{m}$ is equal to zero, we can find an increasing sequence $\left(T_{m}\right)_{m=1}^{\infty}$ in $\mathbb{T}$ such that

$$
\frac{\mu_{\Delta}\left(\left\{t \leq s: f(t) \notin I_{m}\right\}\right)}{\sigma(s)-a}<\frac{1}{m}, \quad s>T_{m} .
$$

Here $a=\min \mathbb{T}$. Let us consider the function $g: \mathbb{T} \rightarrow \mathbb{R}$ defined as follows:

$$
g(t)= \begin{cases}\lambda, & T_{m}<t \leq T_{m+1} \text { and } f(t) \notin I_{m} \\ f(t), & \text { otherwise. }\end{cases}
$$

It is clear that $g$ is a measurable function and $\lim _{t \rightarrow \infty} g(t)=\lambda$. Indeed, for $t>T_{m}$, either $g(t)=\lambda$ or $g(t)=f(t) \in I_{m}$. In this case, $|g(t)-\lambda| \leq 2^{1-m}$ holds.

Finally, we shall show that $f(t)=g(t)$ for $\Delta$-a.a. $t$. For this purpose, consider

$$
\{t \leq s: f(t) \neq g(t)\} \subset\left\{t \leq s: f(t) \notin I_{m}\right\},
$$

$T_{m}<s \leq T_{m+1}$. Thus, from (2.2), we get

$$
\frac{\mu_{\Delta}(\{t \leq s: f(t) \neq g(t)\})}{\sigma(s)-a} \leq \frac{\mu_{\Delta}\left(\left\{t \leq s: f(t) \notin I_{m}\right\}\right)}{\sigma(s)-a}<\frac{1}{m}
$$

which yields $\delta_{\Delta}(\{t \in \mathbb{T}: f(t) \neq g(t)\})=0$, that is, $f(t)=g(t)$ for $\Delta$-a.a. $t$.

(iii) $\Rightarrow$ (i): Let $f(t)=g(t)$ for $\Delta$-a.a. $t$ and $\lim _{t \rightarrow \infty} g(t)=L$. For a given $\varepsilon>0$, we have

$$
\{t \in \mathbb{T}:|f(t)-L| \geq \varepsilon\} \subset\{t \in \mathbb{T}: f(t) \neq g(t)\} \cup\{t \in \mathbb{T}:|g(t)-L| \geq \varepsilon\} .
$$

Since $\lim _{t \rightarrow \infty} g(t)=L$, the second set that appears on the right-hand side of the above inclusion relation is bounded, and thus $\delta_{\Delta}(\{t \in \mathbb{T}:|g(t)-L| \geq \varepsilon\})=0$. In addition, $f(t)=$ $g(t)$ for $\Delta$-a.a. $t$ yields $\delta_{\Delta}(\{t \in \mathbb{T}: f(t) \neq g(t)\})=0$. In conclusion, $\delta_{\Delta}(\{t \in \mathbb{T}:|f(t)-L| \geq$ $\varepsilon\})=0$, namely $\Delta-\lim _{t \rightarrow \infty} f(t)=L$. 
Competing interests

The authors declare that they have no competing interests.

\section{Authors' contributions}

MSS carried out the studies on Functional Analysis, participated theory of statistical convergence and their relations. NOT participated in the measure theory studies and performed the relation convergence with measure and drafted the manuscript.

\section{Acknowledgements}

The editor and referee(s) remark that the results obtained in this paper and many other characterizations (not included here) have already been presented independently with a similar title by C. Turan and O. Duman at the AMAT 2012 International Conference on Applied Mathematics and Approximation Theory, May 17-20, 2012, Ankara-Turkey (http://amat2012.etu.edu.tr) and submitted to the Springer Proceeding.

Received: 14 June 2012 Accepted: 11 September 2012 Published: 2 October 2012

\section{References}

1. Fast, H: Sur la convergence statistique. Colloq. Math. 2, 241-244 (1951)

2. Schoenberg, IJ: The integrability of certain functions and related summability methods. Am. Math. Mon. 66, 361-375 (1959)

3. Fridy, JA: On statistical convergence. Analysis 5, 301-313 (1985)

4. Hilger, S: Ein maßkettenkalkül mit anwendung auf zentrumsmanningfaltigkeiten. PhD thesis, Universität, Würzburg (1988)

5. Guseinov, GS: Integration on time scales. J. Math. Anal. Appl. 285, 107-127 (2003)

6. Cabada, A, Vivero, D: Expression of the Lebesgue $\Delta$-integral on time scales as a usual Lebesgue integral: application to the calculus of $\Delta$-antiderivatives. Math. Comput. Model. 43, 194-207 (2006)

7. Rzezuchowski, T: A note on measures on time scales. Demonstr. Math. 38(1) 79-84 (2005)

8. Deniz, A, Ufuktepe, Ü: Lebesgue-Stieltjes measure on time scales. Turk. J. Math. 33, 27-40 (2009)

doi:10.1186/1029-242X-2012-219

Cite this article as: Seyyidoglu and Tan: A note on statistical convergence on time scale. Journal of Inequalities and Applications $20122012 \cdot 219$

\section{Submit your manuscript to a SpringerOpen ${ }^{\ominus}$ journal and benefit from:}

- Convenient online submission

- Rigorous peer review

- Immediate publication on acceptance

Open access: articles freely available online

High visibility within the field

- Retaining the copyright to your article 